\title{
Inhibition of JNK suppresses autophagy and attenuates insulin resistance in a rat model of nonalcoholic fatty liver disease
}

\author{
HUA YAN $^{1}$, YANQIONG GAO ${ }^{2}$ and YING ZHANG ${ }^{1}$ \\ Departments of ${ }^{1}$ Gerontology and ${ }^{2}$ Functional Examination, \\ Shaanxi Provincial People's Hospital, Xi'an, Shaanxi 710068, P.R. China
}

Received September 21, 2015; Accepted October 3, 2016

DOI: $10.3892 / \mathrm{mmr} .2016 .5966$

\begin{abstract}
Nonalcoholic fatty liver disease (NAFLD) is a common chronic liver disease, the pathological process of which is complex. Activation of the c-Jun N-terminal kinase (JNK) signaling pathway is associated with the mechanism underlying obesity-induced insulin resistance. Furthermore, the JNK signaling pathway and dysfunctional autophagy serve important roles in hepatic lipid metabolism. However, the exact role of JNK in autophagy and obesity-induced insulin resistance is not fully understood. Therefore, the present study aimed to investigate the underlying mechanisms by which the JNK signaling pathway regulates autophagy and insulin resistance in fatty liver. A rat model of NAFLD was established using a high-fat diet (HFD), and insulin resistance in the livers of HFD rats was determined by peritoneal glucose tolerance testing. The results indicated that a HFD induced impaired glucose tolerance, liver function injury, insulin resistance and increased autophagy in rats. Treatment with SP600125, an inhibitor of JNK, relieved NAFLD in rats. Furthermore, SP600125 decreased the expression levels of autophagy-associated genes, including Beclin-1, microtubule-associated protein $1 \mathrm{~A} / 1 \mathrm{~B}$ light chain 3 , autophagy related gene (Atg) 3 and Atg5, and the phosphorylation of insulin receptor (IR) $\beta$-subunit, IR substrate- 1 and protein kinase $B$ in vivo. In conclusion, JNK inhibition may suppress autophagy and attenuate insulin resistance. Therefore, JNK inhibition
\end{abstract}

Correspondence to: Dr Hua Yan, Department of Gerontology, Shaanxi Provincial People's Hospital, 256 West Youyi Road, Beilin, Xi'an, Shaanxi 710068, P.R. China

E-mail: yanh2501@yeah.net

Abbreviations: NAFLD, nonalcoholic fatty liver disease; JNK, c-Jun N-terminal kinase; IR, insulin receptor; HFD, high-fat diet; IR $\beta$, insulin receptor $\beta$-subunit; IRS-1, insulin receptor substrate-1; PI3K, phosphatidylinositol 3-kinase; Akt, protein kinase $\mathrm{B}$; Atg7, autophagy related gene 7; ALT, alanine aminotransferase; AST, aspartate aminotransferase; T-CHO, total cholesterol; TG, triglyceride; FFAs, free fatty acids; TNF- $\alpha$, tumor necrosis factor- $\alpha$; mTOR, mammalian target of rapamycin

Key words: JNK, autophagy, insulin resistance, nonalcoholic fatty liver disease may provide a novel therapeutic strategy for the treatment of NAFLD.

\section{Introduction}

Nonalcoholic fatty liver disease (NAFLD) is currently considered the most common chronic liver disease in developed countries, and is closely associated with insulin resistance and other metabolic risk factors, including central abdominal obesity and dyslipidemia (1). In recent years, the morbidity associated with NAFLD has increased, whereas the age of onset is decreasing. However, the exact pathogenesis of NAFLD remains to be fully elucidated, and at present, there is no specific pharmacological approach to regulate hepatic steatosis.

Due to insulin resistance, elevated insulin stimulates blood sugar uptake and storage through binding to the insulin receptor (IR), which promotes phosphorylation of IR $\beta$-subunit (IR $\beta$ ) and IR substrate-1 (IRS-1), and activation of downstream signaling via phosphatidylinositol 3-kinase (PI3K)-protein kinase $\mathrm{B}$ (Akt) (2,3). C-Jun N-terminal kinase (JNK; including JNK1, JNK2 and JNK3 isoforms) is associated with insulin resistance and is activated by inflammatory cytokines and free fatty acids (FFAs), which are associated with type 2 diabetes $(2,4,5)$. A previous study suggested that JNK activation accelerates lipid accumulation and causes liver injury (6). Furthermore, activated JNK phosphorylates its downstream target, IRS-1, on serine 307 to attenuate insulin sensitivity (7). Sun et al (8) reported that activation of the inositol-requiring protein 1 (IRE1)-JNK pathway is a key factor in impaired hepatic insulin signaling transduction in the livers of mice fed a high-fructose diet (8). In addition, JNK activation is able to upregulate the expression levels of Beclin-1 and microtubule-associated protein 1A/1B light chain 3 (LC3)II in cancer cells, whereas SP600125 (an inhibitor of JNK) or JNK knockdown reversed this process (9). Kluwe et al demonstrated that hepatic fibrosis was modulated by JNK inhibition in hepatic stellate cells and JNK1-deficient mice (10). These findings suggested that JNK signaling may serve a crucial role in the initiation and progression of NAFLD.

Autophagy is a crucial physiological process that has an important role in cellular homeostasis by regulating intracellular lipid stores, and eliminating damaged organelles and misfolded proteins in obesity $(11,12)$. Autophagy has been reported to be implicated in liver physiology and pathogenesis (13). Autophagy dysfunction has been detected in the liver 
from patients with NAFLD and in murine models of NAFLD, as well as in lipid-overloaded human hepatocytes (14-16). A previous study demonstrated that activation of autophagy by rapamycin ameliorated endoplasmic reticulum (ER) stress and decreased apoptosis in human hepatocarcinoma-7 cells (17). Furthermore, inhibition of autophagy using 3-methyladenine markedly increased triglyceride levels in hepatocytes treated with oleate (11). Defective hepatic autophagy in mouse models of obesity also promoted accumulation of fat in the liver and aggravated HFD-induced liver injury. In addition, genetic or molecular suppression of autophagy in various cells promotes ER stress and results in defective IR signaling. Overexpression of autophagy related gene 7 (Atg7) by adenovirus-Atg7 injection was able to alleviate liver condition and insulin resistance in ob/ob mice and in HFD-fed mice (18). These findings suggested that autophagy may serve a protective role in liver injury; however, conflicting results have also been published regarding the function of autophagy in the progression of NAFLD $(11,19)$. A previous study in mice with liver-specific FAK family kinase-interacting protein of $200 \mathrm{kDa}$ deficiency demonstrated that autophagy inhibition prevents lipogenesis and reduces hepatic steatosis (14). Similarly, Kim et al demonstrated that skeletal muscle-specific Atg7 deficiency ameliorated insulin resistance and reduced diet-induced obesity in mice fed a HFD (12). The protective mechanism of autophagy in the pathological process of NAFLD requires further elucidation to provide guidance for autophagy-targeting therapeutic strategies for the treatment of NAFLD.

The JNK signaling pathway is essential for the autophagic process. Activation of JNK contributes to Beclin-1 expression, mediates dysregulated autophagy modulation and mediates p53 phosphorylation (20). Wei et al demonstrated that JNK1-mediated multisite phosphorylation of B-cell lymphoma 2 (Bcl-2) stimulates starvation-induced autophagy by disrupting the Bcl-2/Beclin-1 complex (21). A recent study reported that FFA-stimulated autophagy in INS-1 cells is suppressed by JNK inhibitor II (SB202190 and SB203580). Furthermore, conversion of LC3I to LC3II in INS-1 cells treated with JNK1-targeted small interfering RNA was significantly inhibited compared with in the control group (22). These findings suggested that abnormal autophagy interferes with lipid metabolism, and dysfunctional autophagy may promote the pathogenesis of NAFLD. However, the precise function of autophagy in lipid metabolism remains controversial, since lipolytic and lipogenic functions of autophagy have both been reported.

JNK, autophagy and insulin resistance are all associated with the pathological process of NAFLD; however, the interactive relationships among them are not fully understood. Therefore, understanding the molecular mechanisms by which the JNK signaling pathway mediates lipid-induced metabolic stress will be of great significance for the development of novel treatments for various obesity-associated diseases. In the present study, the relationships among them were illustrated in vivo. A rat model of NAFLD was used to investigate the roles of the JNK signaling pathway in insulin resistance and autophagy. In addition, the present study examined whether the symptoms of NAFLD in rats could be alleviated by inhibition of JNK. The findings of the present study define a core function for JNK in the progression of NAFLD.

\section{Materials and methods}

Animals. Male Sprague Dawley rats (age, 8 weeks; 180-200 g) were obtained from the Animal Center of Xi'an Jiaotong University (Xi'an, China). Rats were maintained under standard conditions: Temperature, $25^{\circ} \mathrm{C} ; 12 \mathrm{~h}$ light/dark cycle; and ad libitum standard laboratory feed and water. Rats were randomly divided into two groups ( $\mathrm{n}=10 /$ group): Normal chow diet (ND) group or HFD group (D12451; 45 kcal\% fat; Research Diets, Inc., New Brunswick, NJ, USA). The rats received the respective diets for 20 weeks. At the end of treatment, rats were anesthetized with sodium pentobarbital (Sigma-Aldrich; Merck Millipore, Darmstadt, Germany; $40 \mathrm{mg} / \mathrm{kg}$ body weight; intraperitoneal injection) were sacrificed by cervical dislocation for biochemical analysis. Muscle, adipose and liver samples from the rats were subjected to western blotting.

Rats fed a HFD were intraperitoneally injected with SP600125 ( $\mathrm{n}=6 ; 30 \mathrm{mg} / \mathrm{kg}$ body weight; Calbiochem; EMD Millipore, Billerica, MA, USA) or phosphate-buffered saline (PBS; $n=6$ ) each day at the end of 20 weeks for an additional 8 weeks. After treatment, the rats were sacrificed by cervical dislocation for biochemical analysis. Liver samples from the rats were subjected to western blotting. The animal experiments were reviewed and approved by the Animal Care and Use Committee of Shaanxi Provincial People's Hospital (Xi'an, China), and were conducted in compliance with Guide for the Care and Use of Laboratory Animals (version 8 in Chinese, 2012).

Biochemical analyses. Serum alanine aminotransferase (ALT), aspartate aminotransferase (AST), total cholesterol (T-CHO) and triglyceride (TG) levels were determined using the cobas automatic analyzer system (Roche Diagnostics, Basel, Switzerland). Blood samples were centrifuged at $1,500 \mathrm{x} g$ for $10 \mathrm{~min}$ at $4^{\circ} \mathrm{C}$. The serum levels of tumor necrosis factor (TNF)- $\alpha$, FFA and insulin were measured using ELISA kits (Assaypro, St. Charles, $\mathrm{MO}, \mathrm{USA}$ ) according to the manufacturer's protocols.

Glucose tolerance and insulin resistance tests. Glucose tolerance tests were conducted after the rats had been fed a ND or HFD for 20 weeks. After $6 \mathrm{~h}$ fasting, rats in the ND and HFD groups were injected intraperitoneally with glucose $(2.0 \mathrm{~g} / \mathrm{kg}$ body weight). Blood was collected from the tail vein at various time points $(0,15,30,60$ and $120 \mathrm{~min})$, and blood glucose levels were measured using a portable glucose meter (Glu-test Sensor; Yuwell-Jiangsu Yuyue Medical Equipment \& Supply Co., Ltd., Nanjing, China). To assess whether insulin resistance occurs in the liver of rats fed a HFD, rats $(n=6)$ were fasted overnight and treated with an intraperitoneal injection of insulin $(1.5 \mathrm{U} / \mathrm{kg})$. After $30 \mathrm{~min}$, the livers were isolated and subjected to western blot analysis for detection of $\mathrm{p}-\mathrm{IR} \beta, \mathrm{p}-\mathrm{IRS}-1$ and $\mathrm{p}-\mathrm{Akt}$.

Western blot analysis. Proteins were isolated from tissues using radioimmunoprecipitation assay lysis buffer (Beyotime Institute of Biotechnology, Haimen, China). The homogenates were centrifuged at $15,000 \times \mathrm{g}$ for $30 \mathrm{~min}$ at $4^{\circ} \mathrm{C}$ and the pellets were discarded. Protein concentration was measured using a BioSpectrometer kinetic spectrometer using the Lowry protein assay kit (Pierce; Thermo Fisher Scientific, Inc., Waltham, MA, USA). A total of $20 \mu \mathrm{g}$ protein was separated by $8 \%$ SDS-polyacrylamide gel electrophoresis, with samples being 
loaded onto each lane of the polyacrylamide gels. Subsequently, proteins in the gels were blotted onto polyvinylidene difluoride membranes (EMD Millipore, Bedford, MA, USA). The membranes were blocked with $5 \%$ nonfat milk in TBS containing $0.1 \%$ Tween-20 at room temperature for $1 \mathrm{~h}$, and were then probed with primary antibodies at $4^{\circ} \mathrm{C}$ overnight. The following primary antibodies were used: Anti-Akt (cat. no. 2920; 1:1,000), anti-phosphorylated (p)-Akt (Ser473) (cat. no. 4060; 1:1,000), anti-Atg3 (cat. no. 3415; 1:1,000), anti-Atg5 (cat. no. 12994; 1:1,000), anti-LC3 (cat. no. 4108; 1:1,000), anti-Beclin-1 (cat. no. 3783; all Cell Signaling Technology, Inc., Danvers, MA, USA); anti-IRS1 (cat. no. 05-784R; 1:2,000), anti-p-IRS1 (Ser307) (cat. no. 05-1087; 1:2,000) (both EMD Millipore); anti-IR $\beta$ (cat. no. sc-711; 1:500), anti-p-IR $\beta$ (Tyr 1150/1151) (cat. no. sc-81500; 1:500), anti-p-JNK1 (Thr183) (cat. no. sc-135642; 1:200), anti-JNK1 (cat. no. sc-571; 1:400) and anti-GAPDH (cat. no. sc-20357; 1:1,000; all Santa Cruz Biotechnology, Inc., Dallas, TX, USA). Blots were then incubated with corresponding horseradish peroxidase-conjugated secondary antibodies (Invitrogen; Thermo Fisher Scientific, Inc.) for 1 hour at room temperature. The blots were visualized using an enhanced chemiluminescence detection system (EMD Millipore) and were exposed to film. Quantification was performed using Image Lab version 2.0 (Bio-Rad Laboratories, Inc., Hercules, CA, USA)

Statistical analysis. The results are presented as the mean \pm standard deviation. Data were analyzed by unpaired Student's t-test. One-way analysis of variance was used to compare the means of $\geq 3$ groups. All data analyses were performed using SPSS v. 17.0 software (SPSS, Inc., Chicago, IL, USA). $\mathrm{P}<0.05$ was considered to indicate a statistically significant difference.

\section{Results}

HFD induces impaired insulin resistance and liver function injury in rats. The body weight of 8-week-old rats fed a ND or HFD was measured every 4 weeks for 20 weeks. After 8 weeks of feeding, the body weight of the HFD-fed rats was markedly higher compared with the ND rats (Fig. 1A). To investigate the effects of NAFLD on insulin resistance, glucose concentration was measured in rats intraperitoneally injected with glucose. After the injection, blood glucose concentration in ND rats increased slightly, but decreased to fasting levels by $120 \mathrm{~min}$. In HFD-fed rats, the blood glucose concentration increased gradually and reached its peak at $60 \mathrm{~min}$. Glucose concentration did not return to normal levels until $120 \mathrm{~min}$ (Fig. 1B). Furthermore, the serum levels of ALT, AST, T-CHO, TG, TNF- $\alpha$, FFA and insulin were markedly higher in HFD-fed rats compared with ND-fed rats (Fig. 1C-F).

HFD induces JNK activation, increased autophagy and insulin resistance in the liver. The JNK pathway is known to be activated by several factors, including oxidative stress, FFAs and TNF- $\alpha$. To investigate the effects of HFD on the JNK pathway, rats were fed a HFD for 20 weeks, and the protein expression levels of p-JNK1 and JNK1 were detected in muscle, adipose and liver samples from the rats. As shown in Fig. 2A, the protein expression levels of $\mathrm{p}-\mathrm{JNK} 1$ were significantly increased in HFD-fed rats compared with in the control group. These results indicate that JNK is activated in muscle, adipose and liver tissues from HFD-fed rats.

To investigate the roles of autophagy in NAFLD, liver samples from rats fed a ND or HFD were subjected to western blotting. Notably, HFD markedly elevated autophagy in the liver samples of the NAFLD rat model, as evidenced by LC3 conversion (LC3 I to LC3 II), and upregulation of Beclin-1, Atg5 and Atg3 protein expression levels (Fig. 2B). These data indicate that autophagy may be upregulated in NAFLD. It may be hypothesized that autophagy signals are associated with the pathogenesis of NAFLD.

To assess whether insulin resistance occurs in the liver of rats fed a HFD, rats were fasted overnight and treated with an intraperitoneal injection of insulin $(1.5 \mathrm{U} / \mathrm{kg})$. After $30 \mathrm{~min}$, the livers were isolated and subjected to western blot analysis. The expression levels of $\mathrm{p}$-IR $\beta, \mathrm{p}$-IRS-1 and p-Akt were significantly higher in HFD-fed rats compared with in the control group , which indicated that HFD increased insulin resistance in the liver of rats (Fig. 2C).

JNK inhibition suppresses autophagy and improves the insulin signaling pathway in HFD rats. A previous study reported that autophagy-mediated insulin receptor downregulation contributes to insulin resistance in vitro and in vivo (23). In addition, JNK has been shown to be implicated in impaired insulin signaling transduction via the IRE1-JNK pathway (8). To investigate the exact role of JNK in the pathological process of NAFLD, the present study examined whether SP600125 was able to reverse pathological events, including abnormal autophagy and decreased insulin sensitivity, in the livers of HFD-fed rats. JNK1 activity was suppressed in rats by SP600125 treatment (Fig. 3A). Subsequently, protein expression levels were detected in rats intraperitoneally injected with SP600125 or PBS, in order to assess changes in autophagy and insulin signaling. The protein expression levels of LC3 II, Beclin-1, Atg5, Atg3, p-IR $\beta$, p-IRS-1 and p-Akt were markedly decreased in the livers of rats treated with SP600125 compared with in the control group (Fig. 3B and C). These results suggest that inhibition of JNK1 phosphorylation may suppress autophagy and improve insulin signaling in a rat model of NAFLD.

SP600125 treatment alleviates symptoms of NAFLD in rats fed a HFD. Serum biochemical indexes in HFD-fed rats were detected following intraperitoneal injection with SP600125 or PBS for an additional 8 weeks. The serum levels of ALT, AST, T-CHO, TG, TNF- $\alpha$, FFA and insulin were significantly decreased in HFD-fed rats treated with SP600125 compared with in the control group (Fig. 4A-D). These results indicate that JNK inhibition may ameliorate NAFLD in HFD-fed rats.

\section{Discussion}

Although previous studies have demonstrated that JNK, autophagy and insulin resistance are correlated, the role of JNK in aberrant autophagy and insulin resistance in the pathogenesis of NAFLD has not yet been addressed. The present study hypothesized that the JNK signaling pathway may be a key modulator of autophagy function in HFD-induced insulin 
A

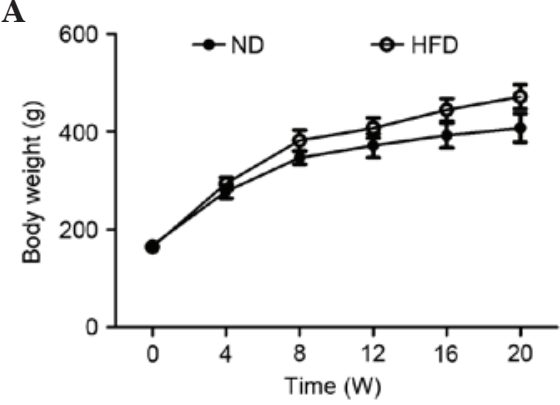

C

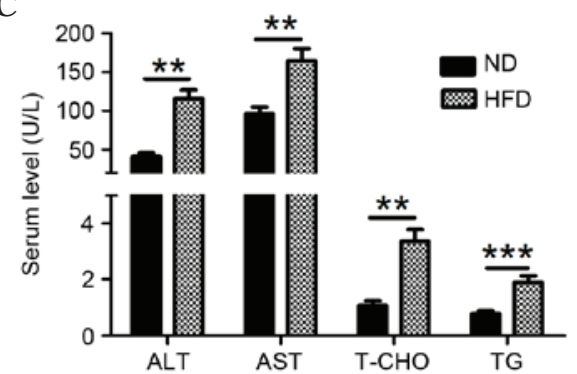

$\mathbf{E}$

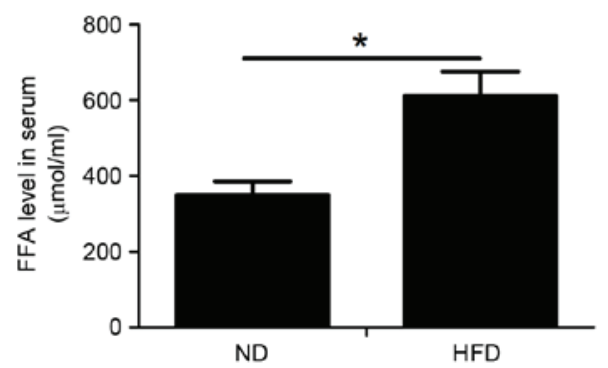

B

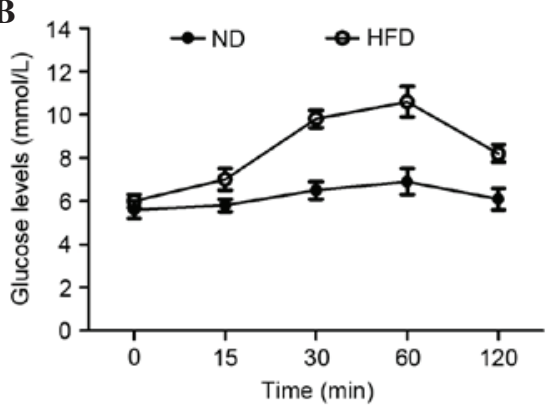

D

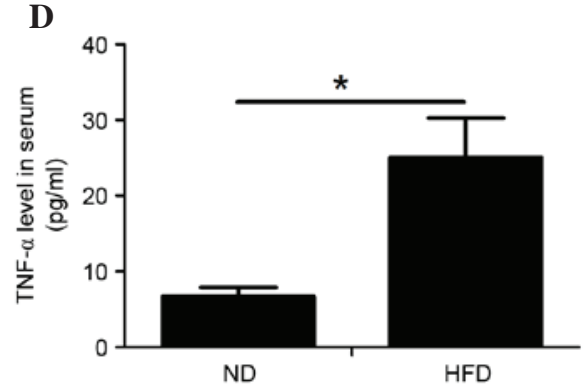

F

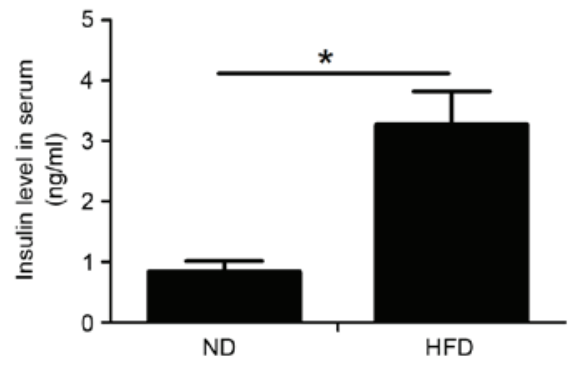

Figure 1. Effects of a HFD on body weight and biochemical indicators of nonalcoholic fatty liver disease. (A) Body weight of rats fed a ND or HFD for 20 weeks. (B) After 20 weeks of feeding, fasting blood glucose levels in the rat models were determined at 0, 15, 30, 60 and 120 min after glucose injection $(2.0 \mathrm{~g} / \mathrm{kg}$ body weight). The serum concentrations of (C) ALT, AST, T-CHO and TG; (D) TNF- $\alpha$; (E) FFA and (F) insulin in rats fed a HFD or ND were detected. Data are presented as the mean \pm standard deviation $(\mathrm{n}=6) .{ }^{*} \mathrm{P}<0.05,{ }^{* *} \mathrm{P}<0.01,{ }^{* * * *} \mathrm{P}<0.001$. HFD, high-fat diet; ND, normal diet; ALT, alanine aminotransferase; AST, aspartate aminotransferase; T-CHO, total cholesterol; TNF- $\alpha$, tumor necrosis factor- $\alpha$; FFA, free fatty acid.

resistance. The results demonstrated that JNK inhibition attenuated insulin resistance and autophagic activity in vivo.

It is widely known that obesity and insulin resistance increase the risk of NAFLD. After years of research, the association between lipids and insulin resistance is widely accepted. Insulin resistance is closely associated with the pathological progression of NAFLD, and JNK is a crucial molecule in the insulin signaling pathway. JNK is activated by almost all forms of metabolic stress, which have been implicated in insulin resistance. Previous studies have suggested that TNF- $\alpha$, FFAs and reactive oxygen species activate $\mathrm{JNK}$, thus promoting initiation and progression of insulin resistance $(24,25)$. In the present study, in HFD-fed obese rats, JNK activity was significantly increased in insulin-sensitive tissues, such as liver and adipose. Activated JNK phosphorylates the serine residues of IRS1, thus contributing to obesity-induced insulin resistance. Furthermore, JNK is critically involved in the promotion of diet-induced fatty liver and metabolic inflammation. JNK1, but not JNK2, has been reported to serve as a crucial molecular link between obesity, metabolic inflammation and disorders of glucose homeostasis (26). Notably, knockout of JNK1 markedly reduced insulin resistance in HFD-fed JNK1-null mice; conversely, mice with JNK2 deficiency fed a HFD were obese and insulin-resistant (27). Özcan et al reported that obesity-induced ER stress may lead to suppression of insulin receptor signaling via hyperactivation of JNK (28). Growing evidence has suggested that JNK may be considered a potential therapeutic target for the treatment of insulin resistance and diabetes. Therefore, elucidating the exact mechanisms by which JNK triggers insulin resistance and results in NAFLD is extremely urgent. To explore this complex pathogenesis, the present study measured JNK activity in the muscle, adipose and liver tissues of rats fed a ND or HFD. Consistent with the results of previous studies, JNK was activated in HFD rats. Furthermore, western blot analysis indicated that autophagy, and IRS-1 and Akt phosphorylation were induced in the liver of rats fed a HFD. The present study focused on the effects of JNK inhibition on autophagy and insulin resistance occurring in NAFLD. 
A

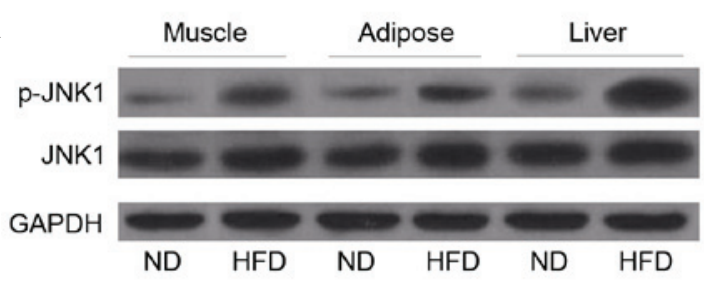

B

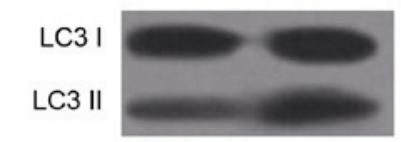

Beclin-1

$\operatorname{Atg} 5$
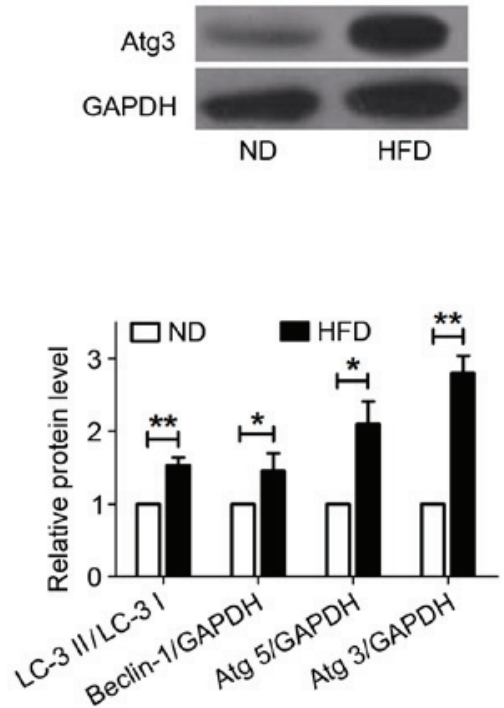

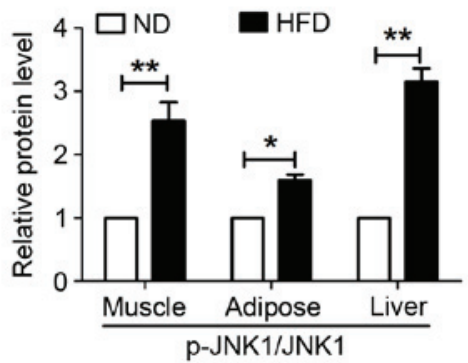

C
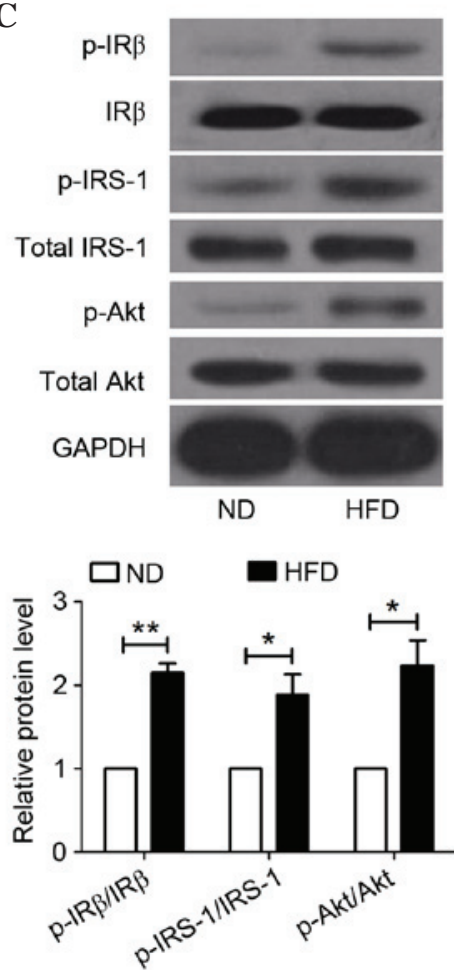

Figure 2. HFD induces JNK activation, increased autophagy and insulin resistance in the liver. (A) Western blot analysis of p-JNK1 and JNK1 in muscle, adipose and liver tissues from a rat model of nonalcoholic fatty liver disease. (B) Autophagy indicators were detected in the liver of rats fed a HFD by western blot analysis. Autophagy levels were assessed by LC3-II/LC3-I ratio, and Beclin-1, Atg5 and Atg3 protein expression. (C) Insulin resistance was examined by western blot analysis using p-IR $\beta$, IR $\beta$, p-IRS-1, total IRS-1, p-Akt and total Akt antibodies. GAPDH was used as a loading control. Data are presented as the mean \pm standard deviation $(n=6)$. ${ }^{*} \mathrm{P}<0.05,{ }^{* *} \mathrm{P}<0.01$. HFD, high-fat diet; ND, normal diet; p-, phosphorylated; JNK1, c-Jun N-terminal kinase 1 ; LC3, microtubule-associated protein 1A/1B light chain 3; Atg, autophagy related gene; IR $\beta$, insulin receptor $\beta$-subunit; IRS, insulin receptor substrate-1; Akt, protein kinase B.

Autophagy is essential for regulating the degradation of lipid droplets when a nutritional deficiency occurs in cells. Autophagic dysfunction may lead to excessive lipid accumulation in the liver, thus contributing to the emergence and progression of NAFLD (29). Autophagy-related gene expression, autophagosomes and the protein levels of autophagy markers have been shown to be significantly increased in obese adipose tissue, along with insulin resistance (30). Conversely, hepatic triglyceride content was increased in rats with an adipose-specific deletion of Atg7 (31). The mammalian target of rapamycin (mTOR) pathway is associated with insulin signaling in several cell lines. A previous study demonstrated that increased activation of mTOR and S6 kinase beta-1 (S6K1) may promote serine phosphorylation of IRS-1, thus resulting in the pathogenesis of hepatic insulin resistance in obese rats (32). Mitochondrial uncoupling protein 2 protects against palmitic acid-induced liver injury by enhancing the function of autophagy (33). The present study demonstrated that serine 307 of IRS-1 was hyperphosphorylated, accompanied by increased p-Akt (Ser473), in HFD-fed rats. Furthermore, activated autophagic flux was observed in the livers of rats fed a HFD. It may be hypothesized that the JNK-mediated autophagy pathway was of great importance in insulin resistance. These results may provide insight into potential NAFLD therapies.

JNK has been reported to regulate autophagy in Drosophila and mammalian cells, in response to various stressors (34). Using a JNK inhibitor and a dominant-negative mutant of JNK, Shimizu et al demonstrated that inactivated JNK can suppress autophagic cell death (20). A previous study demonstrated that autophagy is inhibited by increased insulin via the mTOR signaling pathway (17). Further associations between 
A

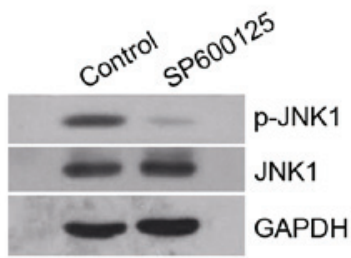

B

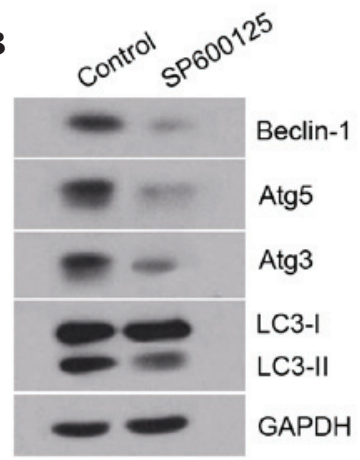

C
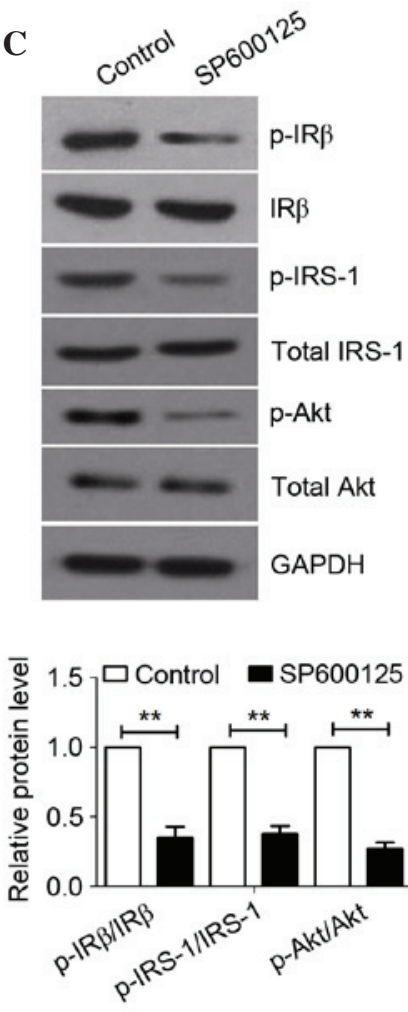

Figure 3. Effects of SP600125 on autophagy and the insulin signaling pathway in HFD-fed rats. After 20 weeks of HFD feeding, the rats were intraperitoneally injected with SP600125 or PBS once a day for 8 weeks, and the rats were then sacrificed. Total protein samples from the livers of HFD rats treated with or without SP600125 were subjected to western blotting. (A) Phosphorylation of JNK was inhibited by SP600125. (B) LC3 conversion, and Beclin-1, Atg5 and Atg3 expression were measured as markers of autophagy. (C) Expression levels of p-IR $\beta$, p-IRS-1 and p-Akt in the liver of rat models were measured to observe changes in insulin resistance. GAPDH was used as a loading control. Data are presented as the mean \pm standard deviation from independent mice ( $\mathrm{n}=6$ ). ${ }^{* *} \mathrm{P}<0.01,{ }^{* * *} \mathrm{P}<0.001$. HFD, high-fat diet; ND, normal diet; $\mathrm{p}-$, phosphorylated; JNK1, c-Jun N-terminal kinase 1; LC3, microtubule-associated protein 1A/1B light chain 3; Atg, autophagy related gene; IR $\beta$, insulin receptor $\beta$-subunit; IRS, insulin receptor substrate-1; Akt, protein kinase B.

A

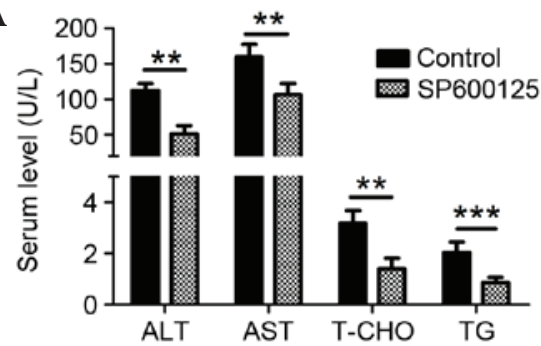

C

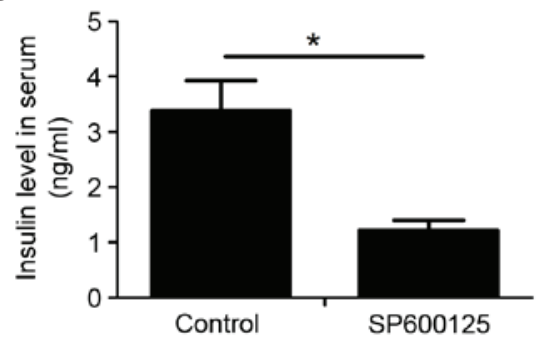

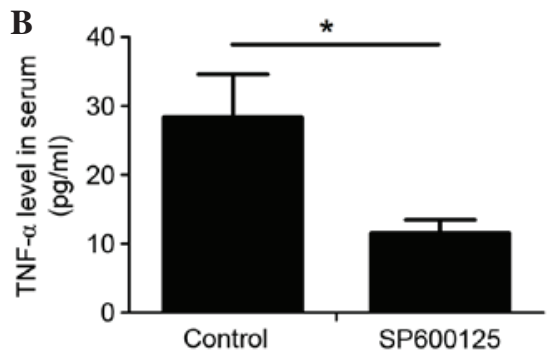

D

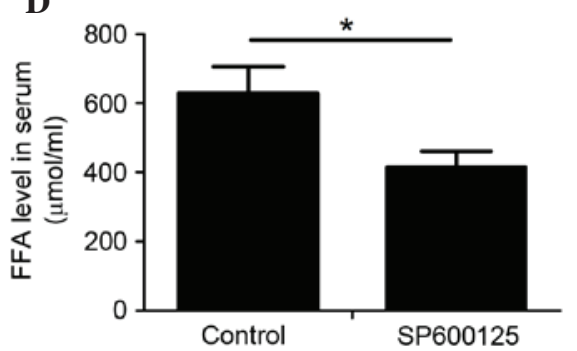

Figure 4. Treatment with SP600125 ameliorated symptoms of nonalcoholic fatty liver disease in HFD-fed rats. The serum levels of (A) ALT, AST, T-CHO and TG; (B) TNF- $\alpha$; (C) insulin and (D) FFA were determined in HFD-fed rats injected with SP600125 or PBS. Data are presented as the mean \pm SD (n=6). ${ }^{*} \mathrm{P}<0.05,{ }^{* *} \mathrm{P}<0.01,{ }^{* * *} \mathrm{P}<0.001$. HFD, high-fat diet; ND, normal diet; ALT, alanine aminotransferase; AST, aspartate aminotransferase; T-CHO, total cholesterol; TNF- $\alpha$, tumor necrosis factor- $\alpha$; FFA, free fatty acid.

dysfunctional autophagy and insulin resistance must be identified. In the present study, the effects of JNK inhibition on autophagy and insulin resistance were detected in HFD-fed rats. The results suggested that JNK inhibition by SP600125 
attenuated autophagy and prevented insulin resistance in the liver of rats fed a HFD. The molecular mechanism by which SP600125 inhibits autophagy and improves insulin resistance in the liver of HFD-fed rats remains to be elucidated.

In conclusion, the present study is the first, to the best of our knowledge, to demonstrate that JNK-mediated autophagy was involved in insulin resistance. Further experiments are required to explore the molecular mechanisms by which p-JNK interferes with autophagy-related gene expression, and subsequently contributes to insulin resistance. The present study also provided evidence suggesting that obesity is closely associated with abnormally elevated JNK1 activity. The results of the present study suggested that selective downregulation of JNK activity presents an attractive opportunity for the treatment of human obesity, insulin resistance, type 2 diabetes and NAFLD. JNK may be considered a potential drug target for the prevention and treatment of NAFLD.

\section{References}

1. Chang E, Park CY and Park SW: Role of thiazolidinediones, insulin sensitizers, in non-alcoholic fatty liver disease. J Diabetes Investig 4: 517-524, 2013.

2. Hirosumi J, Tuncman G, Chang L, Görgün CZ, Uysal KT, Maeda K, Karin M and Hotamisligil GS: A central role for JNK in obesity and insulin resistance. Nature 420: 333-336, 2002 .

3. Brännmark C, Nyman E, Fagerholm S, Bergenholm L, Ekstrand EM, Cedersund G and Strålfors P: Insulin signaling in type 2 diabetes: Experimental and modeling analyses reveal mechanisms of insulin resistance in human adipocytes. J Biol Chem 288: 9867-9880, 2013.

4. Sethi JK and Hotamisligil GS: The role of TNF alpha in adipocyte metabolism. Semin Cell Dev Biol 10: 19-29, 1999.

5. Boden G: Role of fatty acids in the pathogenesis of insulin resistance and NIDDM. Diabetes 46: 3-10, 1997.

6. Schattenberg JM, Singh R, Wang Y, Lefkowitch JH, Rigoli RM, Scherer PE and Czaja MJ: JNK1 but not JNK2 promotes the development of steatohepatitis in mice. Hepatology 43: $163-172,2006$.

7. Hiratani K, Haruta T, Tani A, Kawahara J, Usui I and Kobayashi M: Roles of mTOR and JNK in serine phosphorylation, translocation and degradation of IRS-1. Biochem Biophys Res Commun 335: 836-842, 2005.

8. Sun RQ, Wang H, Zeng XY, Chan SM, Li SP, Jo E, Leung SL, Molero JC and Ye JM: IRE1 impairs insulin signaling transduction of fructose-fed mice via JNK independent of excess lipid. Biochim Biophys Acta 1852: 156-165, 2015.

9. Li DD, Wang LL, Deng R, Tang J, Shen Y, Guo JF, Wang Y, Xia LP, Feng GK, Liu QQ, et al: The pivotal role of c-Jun NH2-terminal kinase-mediated Beclin 1 expression during anticancer agents-induced autophagy in cancer cells. Oncogene 28: 886-898, 2009.

10. Kluwe J, Pradere JP, Gwak GY, Mencin A, De Minicis S, Osterreicher CH, Colmenero J, Bataller R and Schwabe RF: Modulation of hepatic fibrosis by c-Jun-N-terminal kinase inhibition. Gastroenterology 138: 347-359, 2010.

11. Singh R, Kaushik S, Wang Y, Xiang Y, Novak I, Komatsu M, Tanaka K, Cuervo AM and Czaja MJ: Autophagy regulates lipid metabolism. Nature 458: 1131-1135, 2009.

12. Kim KH, Jeong YT, Oh H, Kim SH, Cho JM, Kim YN, Kim SS, Kim do H, Hur KY, Kim HK, et al: Autophagy deficiency leads to protection from obesity and insulin resistance by inducing Fgf21 as a mitokine. Nat Med 19: 83-92, 2013.

13. Rautou PE, Mansouri A, Lebrec D, Durand F, Valla D and Moreau R: Autophagy in liver diseases. J Hepatol 53: 1123-1134, 2010.
14. Ma D, Molusky MM, Song J, Hu CR, Fang F, Rui C, Mathew AV, Pennathur S, Liu F, Cheng JX, et al: Autophagy deficiency by hepatic FIP200 deletion uncouples steatosis from liver injury in NAFLD. Mol Endocrinol 27: 1643-1654, 2013.

15. Li L, Hai J, Li Z, Zhang Y, Peng H, Li K and Weng X: Resveratrol modulates autophagy and NF- $\mathrm{B}$ activity in a murine model for treating non-alcoholic fatty liver disease. Food Chem Toxicol 63: 166-173, 2014.

16. Christian P, Sacco J and Adeli K: Autophagy: Emerging roles in lipid homeostasis and metabolic control. Biochim Biophys Acta 1831: 819-824, 2013.

17. Gonzalez-Rodriguez A, Mayoral R, Agra N, Valdecantos MP, Pardo V, Miquilena-Colina ME, Vargas-Castrillón J, Lo Iacono O, Corazzari M, Fimia GM, et al: Impaired autophagic flux is associated with increased endoplasmic reticulum stress during the development of NAFLD. Cell Death Dis 5: e1179, 2014.

18. Yang L, Li P, Fu S, Calay ES and Hotamisligil GS: Defective hepatic autophagy in obesity promotes ER stress and causes insulin resistance. Cell Metab 11: 467-478, 2010.

19. Shibata M, Yoshimura K, Furuya N, Koike M, Ueno T, Komatsu M, Arai H, Tanaka K, Kominami E and Uchiyama Y: The MAP1-LC3 conjugation system is involved in lipid droplet formation. Biochem Biophys Res Commun 382: 419-423, 2009.

20. Shimizu S, Konishi A, Nishida Y, Mizuta T, Nishina H, Yamamoto A and Tsujimoto Y: Involvement of JNK in the regulation of autophagic cell death. Oncogene 29: 2070-2082, 2010.

21. Wei Y,Pattingre S, SinhaS, Bassik M and Levine B: JNK1-mediated phosphorylation of Bcl-2 regulates starvation-induced autophagy. Mol Cell 30: 678-688, 2008.

22. Komiya K, Uchida T, Ueno T, Koike M, Abe H, Hirose T, Kawamori R, Uchiyama Y, Kominami E, Fujitani Y and Watada H: Free fatty acids stimulate autophagy in pancreatic $\beta$-cells via JNK pathway. Biochem Biophys Res Commun 401: 561-567, 2010.

23. Zhou L, Zhang J, Fang Q, Liu M, Liu X, Jia W, Dong LQ and Liu F: Autophagy-mediated insulin receptor down-regulation contributes to endoplasmic reticulum stress-induced insulin resistance. Mol Pharmacol 76: 596-603, 2009.

24. D'Adamo E, Cali AM, Weiss R, Santoro N, Pierpont B, Northrup V and Caprio S: Central role of fatty liver in the pathogenesis of insulin resistance in obese adolescents. Diabetes Care 33: 1817-1822, 2010.

25. Kodama Y and Brenner DA: c-Jun N-terminal kinase signaling in the pathogenesis of nonalcoholic fatty liver disease: Multiple roles in multiple steps. Hepatology 49: 6-8, 2009.

26. Solinas G and Karin M: JNK1 and IKK beta: Molecular links between obesity and metabolic dysfunction. FASEB J 24: 2596-2611, 2010.

27. Singh R, Wang Y, Xiang Y, Tanaka KE, Gaarde WA and Czaja MJ: Differential effects of JNK1 and JNK2 inhibition on murine steatohepatitis and insulin resistance. Hepatology 49: 87-96, 2009.

28. Özcan U, Cao Q, Yilmaz E, Lee AH, Iwakoshi NN, Ozdelen E, Tuncman G, Görgün C, Glimcher LH and Hotamisligil GS: Endoplasmic reticulum stress links obesity, insulin action and type 2 diabetes. Science 306: 457-461, 2004.

29. Amir M and Czafja MJ: Autophagy in nonalcoholic steatohepatitis. Expert Rev Gastroenterol Hepatol 5: 159-166, 2011.

30. Kovsan J, Blüher M, Tarnovscki T, Klöting N, Kirshtein B, Madar L, Shai I, Golan R, Harman-Boehm I, Schön MR, et al: Altered autophagy in human adipose tissues in obesity. J Clin Endocrinol Metab 96: E268-E277, 2010.

31. Zhang Y, Goldman S, Baerga R, Zhao Y, Komatsu M and Jin S: Adipose-specific deletion of autophagy-related gene 7 (atg7) in mice reveals a role in adipogenesis. Proc Natl Acad Sci USA 106: 19860-19865, 2009.

32. Khamzina L, Veilleux A, Bergeron S and Marette A: Increased activation of the mammalian target of rapamycin pathway in liver and skeletal muscle of obese rats: Possible involvement in obesity-linked insulin resistance. Endocrinology 146: 1473-1481, 2005.

33. Lou J, Wang Y, Wang X and Jiang Y: Uncoupling protein 2 regulates palmitic acid-induced hepatoma cell autophagy. Biomed Res Int 2014: 810401, 2014.

34. Sekine Y, Takeda K and Ichijo H: The ASK1-MAP kinase signaling in ER stress and neurodegenerative diseases. Curr Mol Med 6: 87-97, 2006. 